\title{
3-D Simulations of Protostellar Jets in Stratified Ambient Media
}

\author{
Elisabete M. de Gouveia Dal Pino \\ University of São Paulo, Instituto Astronômico e Geofísico \\ Av. Miguel Stéfano, 4200, São Paulo, SP 04301-901, Brazil \\ dalpino@astro1.iagusp.usp.br \\ Mark Birkinshaw \\ Departmente of Physics \\ University of Bristol, Bristol, UK
}

Received __; accepted _ 


\begin{abstract}
We present fully three-dimensional hydrodynamical simulations of radiative cooling jets propagating into stratified isothermal ambient media with power-law density and pressure distributions. The parameters used are mainly suitable for protostellar jets but results applicable to extragalactic jets are also presented. Comparisons are made with previous simulations of jets through homogeneous media. We find that for radiative cooling jets propagating into regions where the ambient medium has an increasing density (and pressure) gradient, the ambient gas tends to compress the cold, low-pressure cocoon of shocked material that surrounds the beam and destroy the bow shock-like structure at the head. The compressing medium collimates the jet and promotes the development of Kelvin-Helmholtz instabilities which cause beam focusing, wiggling and the formation of internal traveling shocks, close to the head, via pinching along the beam. This remarkably resembles the structure of some observed systems (e.g. Haro 6-5B northern and HH 24G jets). These effects are larger for jets with smaller density ratio between jet and environment $\eta$ (tested for $\eta=1$, 3, and 10) and larger Mach number $M_{a}=v_{j} / c_{a}$ (tested for $M_{a}=12$ and 24, where $v_{j}$ is the jet velocity and $c_{a}$ the ambient sound speed). In an ambient medium of decreasing density (and pressure), the beam is poorly collimated and relaxes, becoming faint. This could explain "invisible" jet sections, like the gap between the parent source and collimated beam (e.g., in HH30 jet). Although, on average, jets propagating into an increasing (decreasing) density environment are decelerated (accelerated) by the increasing (decreasing) ram pressure of the ambient medium, we find that their propagation velocities have an oscillating pattern. The internal traveling shocks that develop in jets propagating into
\end{abstract}


positive density gradient environments display a similar velocity variation, in qualitative agreement with recent measurements of fluctuations in the tangential velocity of the knots of Haro 6-5B jet. Finally, runs of adiabatic jets into similar stratified environments indicate that they are less affected by the effects of stratification than the cooling jets because their higher pressure cocoons are better able to preserve the beam structure.

Subject headings: hydrodynamics - shock waves - stars: early-type - ISM: jets and outflows 


\section{Introduction}

Supersonic jets emanating from protostars and active galactic nuclei propagate into complex media. Protostellar jets, for example, are immersed in molecular clouds which are composed of many smaller dense clouds (e.g. Mundt, Ray and Raga 1991, hereafter MRR; Bally and Devine 1994). Examining a sample of 15 protostellar jets, MRR have found that the flows are often poorly collimated close to the source and become better collimated at large distances from their sources. The Haro 6-5B jet, in particular, after a section of strong expansion, narrows with increasing distance from the source, presumably because of reconfinement. These observations suggest that, in addition to some local collimation near the source, a very large scale collimation mechanism is at work, which is possibly due to the jet external environment (MRR).

The question of what happens to radio-emitting extragalactic jets propagating into a non-homogeneous medium was discussed by Sanders (1983), Falle and Wilson (1985a, b),

Wiita, Rosen and Norman (1990) and Hardee et al. (1992), who performed two-dimensional hydrodynamical simulations of adiabatic, light jets. Since the radiative cooling distance behind the post-shock gas in protostellar jets is smaller than the jet radius, an adiabatic approach is inappropriate. Furthermore, the lack of backflowing cocoons associated with protostellar jets, and the large advance speeds of their working surfaces, suggest they are usually denser than their surroundings. Earlier numerical modeling of jets emanating from protostars were performed for beams propagating into homogeneous ambient media (e.g., Blodin, Fryxell and Königl 1990, hereafter BFK; Gouveia Dal Pino and Benz 1993, hereafter GB93; Gouveia Dal Pino and Benz 1994, hereafter GB94; Chernin et al. 1994, hereafter CMGB; Stone and Norman 1993 (hereafter SN), 1994a, b; Biro and Raga 1994). In this work, we examine the effects of stratified environments on the structure of radiatively 
cooling and adiabatic, initially heavy jets with the aid of three-dimensional numerical simulations using the SPH technique (e.g. GB93). Some of the results of these simulations and their possible correlation with observed wiggling jets have been recently summarized in Gouveia Dal Pino, Birkinshaw and Benz (1995, hereafter GBB). We here describe in more detail the results for a range of environmental and jet parameters.

Previous work of jets propagating into homogeneous ambient media has demonstrated that internal traveling knots may be produced by small-period velocity variations in the ejection of the jet (e.g., Raga et al. 1990, Raga and Kofman 1992, Kofman And Raga 1992, Hartigan and Raymond 1992, GB94, SN). This mechanism favors the formation of knots closer to the driving source which become fainter and disappear at larger distances, as for example, in jet systems like HH34 and HH111. In the present work, we find that the propagation of a radiative cooling jet into an atmosphere of increasing density (pressure) may drive the formation of internal knots closer to the jet head. This seems to be the case, for example, for the Haro 6-5B northern and $\mathrm{HH} 24 \mathrm{G}$ jets.

The organization of this work is as follows. In $\S 2$, based on simple one-dimensional arguments, we discuss the basic theoretical properties of jets propagating into stratified ambient media and describe the assumptions of our numerical model, including the initial and boundary conditions, and the model parameters adopted for the simulations. In $\S 3$, we present the results of our hydrodynamical simulations for jets propagating into stratified ambient media with positive and negative density gradients, and different values of the density ratio between jet and ambient material, $\eta$, and of the Mach number, $M_{a}$. Finally, in $\S 4$, we summarize our results and discuss their possible applications to the observed protostellar jets. 


\section{Description of the Model}

\subsection{Theoretical Grounds}

The basic properties of jets propagating into homogeneous ambient media are described in BFK and GB93. Similarly to Hardee et al. (1992), we assume an initial isothermal ambient medium $\left(T_{a}=10^{4} \mathrm{~K}\right)$ with density (and pressure) distribution stratified along the jet axis $x$ :

$$
n_{a}(x)=n_{a}\left(x_{o}\right) F(x)
$$

where

$$
F(x)=\left[\alpha\left(x-x_{o}\right)+1\right]^{\beta}
$$

and $n_{a}$ is the number density, $x_{o}$ is the value of $x$ at the jet inlet (in units of $R_{j}$, the jet radius), $\alpha$ is a dimensionless parameter of the model and the exponent $\beta= \pm 5 / 3$ for positive and negative density gradients, respectively. Such profiles were chosen for compatibility with previous simulations of jets propagating into homogeneous environments. Also, they are consistent with the observed density distributions of the clouds which involve protostars (e.g., Fuller and Myers 1992). While a negative density gradient may represent,

for example, the atmosphere that a jet encounters when it emerges from the protostar and propagates through the cloud that envelopes the parent source, a positive density gradient may occur when a fully-emerged jet enters an external cloud (GBB) since the density of the cloud increases as the jet submerges. In the simulations, the atmosphere was held steady by an appropriate graviational potential whose effects on the jet dynamics are neglectable since the jets are assumed to be highly supersonic.

A supersonic jet propagating into a stratified ambient gas will develop a shock pattern at its head whose structure and strength will depend on the ambient density profile. The impacted ambient material is accelerated by a forward bow shock whose velocity estimated 
from momentum flux conservation in one-dimensional analysis is approximately given by:

$$
v_{b s} \approx \frac{v_{j}}{1+(\epsilon \eta)^{-1 / 2} F(x)^{1 / 2}}
$$

and the jet material is decelerated at the jet shock with a velocity $v_{j s} \approx v_{j}-v_{b s}$. In the equation above $\eta=n_{j}\left(x_{o}\right) / n_{a}\left(x_{o}\right)$ is the ratio between the jet and ambient number densities at the jet inlet $\left(x=x_{o}\right)$, and $\epsilon^{1 / 2}=R_{j} / R_{h}$ is the ratio of jet to head radius. For a homogeneous ambient medium, for which $F(x)=1$, eq. 3 becomes identical to eq. 2 of GB93. As long as $\epsilon$ remains constant, eq. (3) predicts a global acceleration (deceleration) of the head of a $\eta>1$ jet propagating into a negative (positive) density gradient ambient medium 円.

For shock velocities $v_{s}>90 \mathrm{~km} \mathrm{~s}^{-1}$, the cooling length in the post shock gas behind the shock is given, in units of the jet radius, by (e.g. BFK):

$$
q_{s} \equiv \frac{d_{c o o l}}{R_{j}} \approx 8 \times 10^{16} R_{j}^{-1} v_{s, 7}^{4} n^{-1}
$$

where $n$ is the preshock number density (we have assumed a number density of nuclei $\approx$ $n / 2)$ and $v_{s, 7}=v_{s} / 10^{7} \mathrm{~cm} \mathrm{~s}^{-1}$. Using eqs. 2 and 3 above, the cooling parameter for the bow shock is:

$$
q_{b s} \equiv \frac{d_{\text {cool }}}{R_{j}} \approx 8 \times 10^{16} R_{j}^{-1} v_{j, 7}^{4} n_{a}\left(x_{o}\right)^{-1} \frac{F(x)^{-1}}{\left[1+(\epsilon \eta)^{-1 / 2} F(x)^{1 / 2}\right]^{4}}
$$

and the cooling parameter for the jet shock is:

$$
q_{j s} \approx q_{b s} \epsilon^{-2} \eta^{-3} F(x)^{3} .
$$

\footnotetext{
${ }^{1}$ However, the numerical simulations below show that $v_{b s}$ often develops an oscillating pattern caused by the combined effects of $\epsilon$ and the ambient ram pressure $\left(n_{a} v_{b s}^{2}\right)$ variations; see $\S 4$.
} 
Eqs. (3, 5, and 6) indicate that, as long as $\epsilon$ remains unaltered and $\eta>1$, as the jet propagates downstream into an ambient medium with negative density gradient (decreasing $F(x)$ ), the gas behind the bow shock will become increasingly adiabatic ( $q_{b s}$ increases), while the jet shock will become increasingly radiative $\left(q_{j s}\right.$ decreases with increasing $v_{b s}$ and hence decreasing $v_{j s}$ ). The reverse situation must hold for jet propagation into an ambient medium with positive density gradient.

We note that, for example, in an ambient medium with negative density gradient as $v_{j s}$ decreases with increasing $v_{b s}$, it may eventually become smaller than $90 \mathrm{kms}^{-1}$. In such a case $q_{j s}$ is more correctly evaluated through (e.g., Falle and Raga 1993)

$$
q_{j s} \simeq 0.31\left(\frac{n_{j}}{1000 \mathrm{~cm}^{-3}}\right)^{-1}\left(\frac{v_{j s}}{45 \mathrm{kms}^{-1}}\right)^{-4.7}\left(\frac{R_{j}}{2 \times 10^{16} \mathrm{~cm}}\right)^{-1}
$$

where $n_{j}$ is the jet number density.

The density, $n_{s h}$, of the cold shell that develops in the working surface from the radiative cooling of the shocked gas can be estimated by balancing the ram pressure of the ambient medium and the thermal pressure of the cooled gas:

$$
n_{s h} K T_{s h} \approx \bar{m} n_{a}\left(x_{o}\right) v_{j}^{2} \frac{F(x)}{\left[1+(\epsilon \eta)^{-1 / 2} F(x)^{1 / 2}\right]^{2}}
$$

The right-hand side gives the ambient ram pressure $\left(\rho_{a} v_{b s}^{2}\right), K$ is the Boltzmann constant and $\bar{m}$ is the mean mass per particle which is constant in the case of complete ionization. Therefore, for a jet propagating downstream into a positive-density-gradient-atmosphere, $n_{s h}$ will increase for an $\eta>1$ jet $(\epsilon \sim$ constant $)$, as long as $\left[(\epsilon \eta)^{-1 / 2} F(x)^{1 / 2}\right]$ remains $<1$. However, as $F(x)$ becomes very large $n_{s h}$ will tend to an approximately constant value $\left(n_{s h} \approx \bar{m} n_{a}\left(x_{o}\right) v_{j}^{2} \eta / K T_{s h} \epsilon\right)$.

We note that eqs. 4-8 above apply only in the region close to the axis of symmetry 
of the jet, as pressure gradient effects must be non negligible off the axis. Nonetheless, §3 below indicates that the predictions of the one-dimensional relations above are qualitatively supported by the 3-D simulations.

\subsection{The Numerical Model}

The hydrodynamics equations are solved using a modified version of the threedimensional gridless, Lagrangean smoothed particle hydrodynamics (SPH) code described in Benz (1990), GB93, GB94 and CMGB. The ambient gas is represented by a 3-D rectangular box filled with particles. A supersonic jet of radius $R_{j}$ is injected continually into the bottom of the box, which has dimensions up to $\sim 30 R_{j}$ in the $\mathrm{x}$-axis and $12 R_{j}$ in the transverse directions y and $\mathrm{z}$. All distances are normalized to the jet radius. The boundaries of the box are assumed to be continuous $(\partial v=0)$ - particles are removed from the system whenever they cross the boundaries. (In the original code (GB93), the transverse boundaries were assumed to be periodic. With the present modification to continuous boundaries, the formation of undesirable reflective waves on the transverse boundaries, later on the simulations, is prevented.) The SPH particles are smoothed out in space with a spherically symmetric kernel function of initial width 0.4 or 0.5 and 0.2 or $0.25 R_{j}$ for the ambient and jet particles, respectively.

As in the previous works (GB94, GB94, and CMGB), the jet and the ambient gas are treated as a single ionized fluid with a ratio of specific heats $\gamma=5 / 3$ and an ideal gas equation of state $p=u(\gamma-1) \rho$, where $u$ is the internal energy per unit mass, $p$ is the thermal pressure, and $\rho=n \bar{m}$ is the mass density.

The radiative cooling (due to collisional excitation and de-excitation, and recombination) is calculated using the cooling function evaluated by Katz (1989) for 
a gas of cosmic abundances cooling from $T=10^{6} \mathrm{~K}$. The cooling is suppressed below $T \approx 10^{4} \mathrm{~K}$ when the assumption of completely ionized flow breaks down and the effects of transfer of ionizing radiation become important. A time-implicit scheme combined with Newton-Raphson (NR) iterations is used to evolve the cooling rate. To monitor accuracy, the maximum change in a timestep is kept less than 30\%. If this condition is not achieved, or if the system fails to converge in $100 \mathrm{NR}$ iterations, the timestep is reduced (to $\Delta t / 2)$ and a solution is once again pursued. The limitations of the cooling assumptions are discussed in GB93 and GB94. By not taking into account the effects of nonequilibrium ionization of the gas or the transfer of ionizing radiation, we are probably underestimating the cooling rate in some parts of the postshock regions by as much as an order of magnitude (e.g., Innes, Giddings, \& Falle 1987). However, a comparison of our results (see also GB93) with multidimensional calculations for steady flows in a homogeneous ambient medium, which have included a nonequilibrium time-dependent cooling $(\mathrm{SN})$, show that the essential dynamical features do not change significantly under the presence of nonequilibrium ionization effects.

The evolution of the system is parameterized by the dimensionless numbers: i) $\eta=n_{j} / n_{a}$ (the ratio between the input jet and ambient number densities at $x_{o}$ (eq. 2); (ii) $M_{a}=v_{j} / c_{a}$ (the initial ambient Mach number, where the ambient sound speed is given by $c_{a}=\left(\gamma k T_{a} / \bar{m}\right)^{1 / 2}$, with $T_{a}$ being the ambient temperature (held constant even in stratified atmospheres) and $\bar{m} \simeq 0.5 m_{H}$ the mean mass per particle for a fully ionized gas of cosmic abundances); (iii) $k_{p}=p_{j} / p_{a}$ (the input pressure ratio which has been assumed to be initially equal to unity in all simulations); and (iv) $q_{b s}(0)$ (eq. 5). The stratification of the ambient medium described by eqs. 1 and 2 adds two new parameters to the models, $\alpha$ and $\beta$.

Based on typical conditions found in protostellar jets, the parameters of the simulations 
were chosen to be the following: density ratio $\eta \simeq 1-10$ (e.g., Mundt, Brügel \& Buhke 1987, Morse et al. 1992, Raga \& Noriega-Crespo 1993); ambient number density $n_{a}(0)$ $\simeq 200 \mathrm{~cm}^{-3}$; jet velocities $v_{j} \simeq 200,400 \mathrm{~km} \mathrm{~s}^{-1}$ (e.g., Reipurth 1989a, Reipurth et al 1992); initial ambient Mach number $M_{a}=12$, 24; jet radius $R_{j}=2 \times 10^{15} \mathrm{~cm}(e . g$. , Raga 1993). For the parameters of the stratified ambient medium (eqs. 1 and 2) we assume $\alpha=0-0.5$ and $\beta= \pm 5 / 3$. We note that the values adopted for $\alpha$ and $\beta$, correspond to

large pressure and density gradients, which allow us to investigate structural effects on the simulated beams without having to make them propagate too many jet radii. In fact, large gradients do exist in the ISM through which the jets propagate, as we have remarked in $\S 1$, and the evolution of these gradients with time is much slower than the evolution of the jet structures, so that the assumption of a nearly steady atmosphere is reasonable.

The limitations to our SPH simulations are discussed in GB93, GB94 and CMGB, in particular, we remark that turbulence which is active in these flows since the Reynolds numbers are very high, $>10^{4}$ (GB93), is difficult to study because our initial particle spacing is quite large relative to the size of eddies that may develop in the flow and the numerical viscosity of the code may be too dissipative. Thus, for this work we can only consider the bulk properties of the flow, i.e., over a size scale larger than that of most of the largest eddies where the internal turbulent motions are averaged out.

\section{The Simulations}

We have performed runs of radiative cooling, supersonic jets using different values of $\eta, M_{a}$, and the $\alpha$ and $\beta$ parameters for the stratified ambient medium. The effects of stratification on adiabatic jets were also examined. The subsections below present the results of these calculations and Table 1 summarizes the values of the input parameters of 
our simulations.

\section{1. $\quad$ Effects of ambient media with different $(\alpha, \beta)$}

Fig. 1 depicts the central density contours and the velocity distribution of five cooling jets at a time $t=0.85$ (in units of $t_{j a}=R_{j} / c_{a}=38.2 \mathrm{yrs}$ ) evolving through ambient media with: a) and b) positive density stratification; c) no stratification; and d) and e) negative density stratification (see Table 1). All the jets have the initial conditions: $\eta=3$, $n_{a}\left(x_{o}\right)=200 \mathrm{~cm}^{-3}, R_{j}=2 \times 10^{15} \mathrm{~cm}, v_{j}=398 \mathrm{~km} \mathrm{~s}^{-1}, M_{a}=24, q_{b s}\left(x_{o}\right) \approx 8.1$ (corresponding

to an initial bow shock velocity $v_{b s} \approx 252 \mathrm{~km} \mathrm{~s}^{-1}$ ), $q_{j s}\left(x_{o}\right) \approx 0.3$ (corresponding to an initial jet shock velocity $v_{j s} \approx 146 \mathrm{~km} \mathrm{~s}^{-1}$ ). The maximum density reached by the cold shell that develops at the head of the jet from the cooling of the shock-heated gas, $n_{s h} / n_{a}\left(x_{o}\right)$, is in rough agreement with eq. 8 . We clearly see an acceleration of the jet due to the decrease of the ambient density and ram pressure $\left(n_{a} v_{b s}^{2}\right)$ from Figs. 1a to 1e. Consistent with eq. (3), the propagation velocity of the beam, $v_{b s}$, increases with increasing $\eta$.

Figs. 2, 3 and 4 compare the density and velocity evolution of three cases with $\eta=3$. In Fig. 2, the ambient medium is homogeneous $(\alpha=0, \beta=0)$; in Fig. 3, the ambient medium has positive density gradient $(\alpha=0.5, \beta=5 / 3)$; and in Fig. 4 , it has negative density gradient $(\alpha=0.5, \beta=-5 / 3)$. The initial conditions are the same as in Fig. 1 .

In the homogeneous medium (Fig. 2), we identify the same features detected in previous works (e.g., BFK, SN, GB93, GB94, CMGB). At the head of the jet, a dense shell of shocked jet material develops. It becomes Rayleigh-Taylor (GB93) unstable and breaks into blobs. The density of the shell also undergoes variations with time due to global thermal instabilities of the radiative shock (see GB93 for details). A thin, low density cocoon containing post-shock jet gas is deposited around the beam. A shroud of shocked 
ambient gas envelops both the beam and the cocoon.

In the increasing density (and pressure) medium (Fig. 3), the cocoon/shroud is compressed and pushed backwards by the increasing ram pressure of the ambient medium (see also GBB). An elongated structure (Fig. 3c, d) replaces the bow shock seen in front of the head of the jet propagating into the uniform medium of Fig. 2. The increasing pressure and density of the surrounding medium collimate the beam and promotes the development of the Kelvin-Helmholtz (K-H) instability (e.g. Birkinshaw 1991), and the inherent discreteness of the SPH code in the beam excites pinch and helical modes (Fig. 3c). These modes over-confine the beam, drive internal shocks, and cause some jet wiggling (see also Figs. 7a, b, and 8 below). It is interesting to note that this beam structure is similar to the one found for adiabatic jets propagating into homogeneous ambient medium (GB93). In the latter cases, however, the collimation of the beam is a result of the hot, high pressure, post shock jet gas in the cocoon immediately behind the working surface.

On average, jets propagating into an ambient medium of increasing density gradient are decelerated by the increasing ambient ram pressure $\left(n_{a} v_{b s}^{2}\right)$. However, for the jet of Fig. 3 (see also Figs. 7 and 8 below), we find that the propagation velocity, $v_{b s}$, has an oscillating pattern caused by competition between the $F(x)$ and $\epsilon(x)$ terms in eq. 3. Since $F(x)$ increases with distance, $v_{b s}$ initially decreases as the jet propagates downstream with $\epsilon^{1 / 2} \approx 1$. As the jet head is compressed by the ambient medium, however, the increase of $\epsilon$ more than compensates for the decrease of $F(x)$ and the jet head accelerates as it narrows. Eventually, the further decrease of $F(x)$ again dominates and the working surface decelerates again. Table 2 shows the velocity variations in the head of the jet of Fig. 3 as a function of the time and the position of the bow shock $\left(x_{b s}-x_{o}\right)$ (see also GBB). The varying bow shock and jet shock velocity $\left(v_{j s} \approx v_{j}-v_{b s}\right)$ may cause the ambient ram pressure $\left(n_{a} v_{b s}^{2}\right.$; Table 2$)$ and the radiative cooling distances behind both shocks, $q_{b s}$ and 
$q_{j s}$, to fluctuate (eqs. 5 and 6 ), and the working surface to become thermally unstable (GB93). On average, however, the bow shock becomes more radiative and the jet shock more adiabatic as the jet propagates downstream, in qualitative agreement with eqs. 5, 6 and 7. Following the oscillating pattern, the maximum density in the shell at the head $n_{s h} / n_{a}\left(x_{o}\right)$ varies from: $\approx 847\left(t / t_{j a}=0.85\right)$, to $3550\left(t / t_{j a}=1.25\right), 651\left(t / t_{j a}=1.65\right)$, and $741\left(t / t_{j a}=1.95\right)$, in Figs. 3a, b, c, and d, respectively. The internal shocks of the beam have peak separations $\sim 1-2 R_{j}$ and travel downstream with a similar variable velocity as the working surface. The shocks closer to the jet head have their speed closer to $v_{b s}$.

In the decreasing density (and pressure) medium (Fig. 4), the jet retains a welldeveloped bow shock and cavity structure. The beam shows a slight decollimation close to the head $\left(\epsilon^{1 / 2} \approx 0.7\right.$ in Figs. 4c, and $\mathrm{d}$; see also Fig. 9). On average, it propagates downstream with increasing velocity, $\left(v_{b s} \approx 285,325,353\right.$, and $376 \mathrm{~km} \mathrm{~s}^{-1}$, in Figs. $4 \mathrm{a}, \mathrm{b}$, c, and d, respectively), due to the declining ambient ram pressure, in rough agreement with eqs. 1, 2, and 3. The cold shell at the head is thin and without fragmentation. No internal shocks are formed along the beam. For an ambient medium with negative density gradient (increasing $\eta$ ), the cooling length behind the bow shock $\left(q_{b s}\right)$ increases as the jet propagates downstream (eqs. 4, 5): $q_{b s}$ increases from 8.1 at $\mathrm{t}=0$ to $\sim 3958$ at $\mathrm{t}=1.45 t_{j a}$, so that the bow shock becomes more adiabatic as the beam propagates. By contrast, $q_{j s}$ initially decreases from 0.3 at $\mathrm{t}=0$ to $\sim 0.1$ at $\mathrm{t}=0.25 t_{j a}$, in agreement with eq. 6 , then increases to $\sim 149$ at $\mathrm{t}=1.45 t_{j a}$ (eq. 7 ), so that the jet shock becomes less radiative.

For comparison, Figs. 5a, b, and c show the mid-plane radiative emissivity $\left(L=n^{2} \Lambda(t)\right)$ contours of the jets of the Figs. 2, 3, and 4, respectively, when their working surfaces are at a position $x \simeq 25 R_{j}$. In the decreasing density ambient medium (Fig.5c), the jet is very faint with a maximum emissivity which is $\sim 1.3 \times 10^{-2}$ of that of the jet in the homogeneous ambient medium (Fig. 5a). The latter, in turn, has a maximum emissivity which is 
$\sim 1.6 \times 10^{-2}$ of that of the jet in the increasing density ambient medium (Fig. 5b). The time evolution of the radiative emissivity of the head of the jets of Figs. 2, 3, and 4 in the axis $(y=z=0)$ is depicted in Fig. 6. As the jet in the decreasing density ambient medium ( $\alpha=0.5, \beta=-5 / 3)$ propagates downstream the emissivity (which is generally dominated by the jet shocked material) decays and the shock becomes mostly adiabatic. For the jet propagating into the homogeneous ambient medium $(\alpha=0, \beta=0)$ the varying pattern of the emissivity is correlated with the density oscillations of the cold shell discussed above. The emissivity of the jet propagating into the increasing density medium $(\alpha=0.5, \beta=5 / 3)$ (which is initially dominated by the bow shock and after $\mathrm{t} \simeq 1.25 t_{j a}$ by the internal knot closest to the head) increases with time, as expected.

\subsection{Jets with different density ratio relative to the ambient medium $\eta$}

Fig. 7 depicts the velocity distribution of the central slice of cooling jets with different $\eta$ propagating into an $\alpha=0.5$ ambient medium with positive $(\beta=5 / 3)$ density (and pressure) stratification (see the corresponding density maps in Fig. 2 of GBB). The jets have for $\eta=1: q_{b s}\left(x_{o}\right) \approx 3.1 ; q_{j s}\left(x_{o}\right) \approx 3.1 ; \eta=3: q_{b s}\left(x_{o}\right) \approx 8.1, q_{j s}\left(x_{o}\right) \approx 0.3 ;$ and $\eta=10$ : $q_{b s}\left(x_{o}\right) \approx 16.7, q_{j s}\left(x_{o}\right) \approx 0.008$, and other initial conditions the same as in previous runs (Table 1 ). The $\eta=1$ jet is the most evolved, reaching the end of the computation domain at $t=1.85 t_{j a}$, while the $\eta=3$ jet reaches the end at $t=1.65 t_{j a}$ and the $\eta=10$ jet at $t=1.25 t_{j a}$. The maximum density of the shells at the jet heads are $n_{s h} / n_{a}\left(x_{o}\right) \approx 211$, 638, and 2540, respectively. Jets with larger $\eta$ are less affected by the increasing ambient density and pressure gradients, and show less focusing by the compressing ambient medium. Consistently, the development of the K-H modes (see also Fig. 3), which cause the beam pinching and wiggling, are stronger on small- $\eta$ jets. The internal oblique shocks driven by 
beam pinching have a typical separation of a few jet radii, in agreement with the observed internal knots of Young Stellar Object jets (e.g., MRR). Like the $\eta=3$ jet (see Fig. 3), both the $\eta=1$ and $\eta=10$ jets also show oscillatory propagation velocities due to the competing effects of the increasing ambient density and the ratio $\epsilon^{1 / 2}$ (eq. 3). Tables 3 and 4 show the velocity variations in the heads of the $\eta=1$ and 10 jets. In the case of the $\eta=10$ jet, the velocity oscillations are smoother, and the smaller velocity variation in this case is not enough to cause significant fluctuations on the ambient ram pressure $\left(n_{a} v_{b s}^{2}\right)$, which decreases with increasing distance. The internal shocks of Figs. 7a, and b travel downstream under a similar variable velocity pattern as the working surface.

Fig. 8 shows that later $\left(t=2.05 t_{j a}\right)$, the knots close to the head of the $\eta=1$ jet break and separate, but this effect may have been amplified by the nearby continuitive boundary.

Fig. 9 depicts three cooling jets with different $\eta$ propagating into an $\alpha=0.5$ ambient medium with negative $(\beta=-5 / 3)$ density (and pressure) stratification and the same initial conditions as Fig. 7. As in Fig. 4, in all cases the cocoon becomes broad and relaxed. The cold shell is thin and its density is low (see Fig. 9), in qualitative agreement with eq. 7. The beam decollimation is significantly larger for the smallest $\eta$ jet.

\subsection{Jets with different $\mathbf{M}_{a}=v_{j} / c_{a}$}

Fig. 10 depicts an $\eta=3$ cooling jet propagating into an $\alpha=0.5$ ambient medium with positive $(\beta=5 / 3)$ density (and pressure) stratification and $M_{a}=12$ (or $v_{j}=199 \mathrm{~km}$ $\mathrm{s}^{-1}$ ), which can be compared with the jet of Fig. 3 for which $M_{a}=24$. The initial values of $q_{b s}\left(x_{o}\right) \approx 0.5$ (eq. 5) and $q_{j s}\left(x_{o}\right) ; \approx 1.9 \times 10^{-2}$ (eq. 7) for $\eta=3$ (Table 1 ) imply that both shocks initially cool very fast and the jet shock is nearly isothermal (e.g. GB93). The jet reaches the end of the domain at $x=20 R_{j}$ at $t=3.85 t_{j a}$ (Fig. 10c). This snapshot can 
be compared with Fig. $3 \mathrm{c}$ where the $M_{a}=24$ jet has propagated about the same distance (at $\mathrm{t}=1.65 t_{j a}$ ). The smaller $M_{a}$ jet seems to be less affected by the focusing effect of the ambient medium with increasing pressure, but the interface with the cocoon is more affected by turbulent entrainment (CMGB).

Fig. 11 depicts a jet propagating into an $\alpha=0.5$ ambient medium with negative ( $\beta=-5 / 3)$ density (and pressure) stratification (compare this $M_{a}=12$ jet with the $M_{a}=24$ case in Fig. 4). The beam decollimation close to the head caused by the decreasing ambient pressure is slightly larger in the smaller, more evolved $M_{a}$ jet. The maximum density in the cold shell at the jet head is also smaller in this latter case.

\subsection{Adiabatic versus Radiative Cooling Jets}

Fig. 12 depicts the central density contours and velocity distribution of an adiabatic and a radiative cooling jet (as in Fig. 3), both with $\eta=3$ and $M_{a}=24$ propagating into an $\alpha=0.5$ ambient medium with positive $(\beta=5 / 3)$ density (and pressure) stratification at $t=1.45 t_{j a}$. The ambient medium compresses the cooling jet more than the adiabatic jet, and the thinner, lower-pressure cocoon around the cooling jet is less able to preserve its structure. The propagation velocities are very similar until $t \approx 1.45 t_{j a}$, when, due to the larger focusing $\left(>\epsilon^{1 / 2}\right)$ of the head, the radiative jet propagates about twice as fast. The maximum density behind the shock is $n_{s h} / n_{a}\left(x_{o}\right) \approx 650$ for the cooling jet and 6 for the adiabatic jet.

Fig. 13 depicts the density contours and velocity distribution of an adiabatic and a radiative cooling jet (also in Fig. 4), both with $\eta=3$ and $M_{a}=24$ propagating into an $\alpha=0.5$ ambient medium with negative $(\beta=5 / 3)$ density (and pressure) stratification, at $t=0.85 t_{j a}$ when they reach $\mathrm{x} \approx 20 R_{j}$. The propagation velocity $v_{b s}$, the decreasing ambient 
ram pressure, and the head decollimation are very similar in both jets, although slightly larger in the adiabatic case. The density behind the shock is: $n_{s h} / n_{a}\left(x_{o}\right) \approx 32$ for the cooling jet and 6 for the adiabatic jet.

\section{Discussion and Conclusions}

In this work, we have presented fully 3-D hydrodynamical simulations of initially overdense, radiative cooling jets propagating into stratified isothermal ambient media with a power-law density and pressure distribution (eq. 1). We also have investigated the behaviour of adiabatic jets propagating into similar stratified environments. As in our previous work (GB93, GB94, CMGB94, GBB), the simplified treatment of the radiative cooling of the gas prevents us from performing a detailed comparison of our models with

the radiation from observed protostellar jets. In particular the density contour maps, while describing reasonably well the expected gas distribution do not necessarily correspond to the observed emission line images. Nonetheless, with the help of those maps, we can delineate some basic structural characteristics and dynamics, and compare with observed protostellar jets.

Our results show that jets propagating into regions of an ambient medium with negative density (pressure) gradient develop a broad and relaxed cocoon and suffer beam decollimation at the head. Their propagation velocity increases, on average, as they propagate downstream due to the drop of the ambient density and ram pressure. The beam decollimation is larger for the small- $\eta$ jets as their momentum flux is smaller (Figs. 4 and 7). No internal knots are formed in these cases and the weak radiative shocks at the head provide little radiation. This suggests a possible explanation for the invisible portions of the observed outflows, as we have proposed in GBB95. For example, the observed gaps close 
to the source of Haro 6-5B (or FS Tau B), AS 353, or 1548 C27 jets (e.g. MRR; Hartigan, Mundt, and Stocke 1986; Mundt et al. 1984, 1987), or the gaps between the beam and the distant bow shock (e.g. Haro 6-5B, ) could be a product of such an effect (see also GBB).

Jets propagating into an ambient medium with increasing density (pressure) have their cocoon/shroud compressed and pushed backwards by the ambient ram pressure and the beam is highly collimated. The compressing medium promotes beam focusing and wiggling, and the formation of traveling internal shocks via beam pinching. The bow shock like structure at the head disappears. Jets with larger $\eta$ are less focused by the compressing medium, as a consequence of their larger momentum flux. We found that within $\sim 30 R_{j}$ a $M_{a}=24\left(v_{j} \approx 398 \mathrm{~km} \mathrm{~s}^{-1}\right)$ jet with $\eta \leq 3$, propagating into a positive density gradient medium wiggles and develops regularly-spaced traveling knots close to the head (see also GBB).

Protostellar jets are generally well collimated and knotty, and often show one or more bow shock features at the head (e.g. Reipurth 1989a; Hartigan, Raymond, and Meaburn 1990). A number of authors (e.g. Hartigan and Raymond 1992, Kofman and Raga 1992, Raga and Kofman 1992, SN; GB94) have shown that the internal traveling bright knots may be produced by small-period variations in the velocity of jet ejection. This mechanism favors the formation of knots closer to the driving source which become fainter and disappear at larger distances, as for example, in jet systems like HH34 and HH111 (e.g. Reipurth 1989b). On the other hand, BFK and GB93 have shown that due to radiative cooling, $\mathrm{K}-\mathrm{H}$ instabilities were less effective at driving internal shocks in beams propagating into a homogeneous ambient medium. In the present work, however, we find that propagation of a radiative cooling jet into an ambient medium of increasing density (pressure) may drive the formation of internal knots close to the jet head. This seems to be the case, for example, for the Haro 6-5B northern and HH 24G jets (Mundt et al. 1990, 
MRR), which present an elongated wiggling and knotty structure far from the source, very similar to those of Figs. 3 and 7 (see GBB for a detailed discussion).

Large amplitude side-to-side wiggles and knots are also observed in the HH83, HH84, HH85, HH110 (e.g., Reipurth 1989a, b), and HH30 jets (Mundt et al 1990, MRR), all of which are immersed in regions. The HH30 jet, for example, belongs to the HLTau/HH30 region which shows five outflows and a number of reflection nebulae associated with YSO's. As for Haro 6-5B jet, we suggest that the observed collimated, wiggling, and knotty structure of these jets could be due to $\mathrm{K}-\mathrm{H}$ instabilities driven by a compressing medium of increasing density (as in Figs. 3 and 7). An alternative, recently discussed by Raga, Cantó, and Biro (1993) is that the jet is ejected from a precessing source into a homogeneous ambient medium.

Also, our results indicate that increasing density environments destroy the bow shock at the head. Some authors have demonstrated that bow shocks can be formed in jets with continuous ejection (e.g., Norman et al. 1982; BFK; GB93) or with multiple outflow episodes of long period (e.g., Hartigan and Raymond 1992, Raga and Kofman 1992; SN; GB94) and can keep their structure stable in portions of the jet where the ambient medium is more homogeneous (GB93). However, there are cases which show no evidence of a terminal bow shock feature (e.g. some sources of the MRR sample), which may match some of our results (Figs. 3, 7, and 8). There are other cases (e.g. Haro 6-5B (MRR), and HH30 jets (López et al. 1995) in which the elongated beam is separated by a long gap from the distant bow shock. The bow shock in these cases could be a relic of an earlier outflow episode, or the place where the jet impacts a dense portion of the ambient medium after a long expansion through a decreasing density (pressure) medium (see GBB).

Although, on average, the jets propagating into an increasing (decreasing) density 
environment are decelerated (accelerated) by the increasing (decreasing) ram pressure of the ambient medium, we find that their propagation velocities $v_{b s}$ have an oscillating pattern due to the competing effects between the varying density ratio $\eta$ and the ratio of jet to head radius $\epsilon^{1 / 2}=R_{j} / R_{h}$. The internal traveling shocks that develop in jets propagating into positive density gradient environments (Figs. 3, 7, and 8) also follow a variable velocity pattern, with their speeds getting closer to $v_{b s}$ as they approach the jet head. This is in qualitative agreement with recent measurements of the tangential velocity of the knots of Haro 6-5B (Eislöfel 1993). Variations of the shock velocity must be accompanied by variations in the intensity of emission from cooling regions behind the shocks. Thus, further detections of intensity and knot proper motion variability in other systems would provide a possible check of our predictions.

In the comparison of jets with different initial Mach numbers $M_{a}$ propagating into ambient portions of increasing density, we find that (Figs. 3 and 10) the smaller $M_{a}$ jet $\left(M_{a}=12\right)$ seems to be less affected by the focusing effect of the ambient medium with increasing pressure. Due to its smaller pressure cocoon, the interface between the cocoon and the smaller $M_{a}$ jet is more affected by lateral turbulent entrainment (e.g. Raga, Cabrit and Cantó 1994; Gouveia Dal Pino 1995). In a recent investigation of radiative cooling jets propagating into homogeneous ambient media (CMGB), we found that the lateral entrainment of ambient gas through turbulent mixing at the contact discontinuity is relevant only for low Mach number $\left(M_{j}=M_{a} \eta^{1 / 2} \leq 3\right)$, low density ratio $(\eta \leq 3)$ flows which, in general, are not appropriate for protostellar jets for which $10<M_{j}<40$ and $\eta>1$. Entrainment of ambient gas seems to be mostly through the bow shock in protostellar jets (CMGB). These results have ruled out models which proposed that the molecular outflows associated with protostellar jets are produced predominantly through turbulent entrainment (e.g., Stahler 1993). In the present study, however, we find that 
jets with $M_{j}=21$ ( or $M_{a}=12$ and $\eta=3$ ) in an ambient medium of increasing density suffer considerable entrainment along the beam. This result suggests that even in high Mach number jets, turbulent entrainment may play an important role in driving molecular outflows if the ambient medium has regions of varying density (and pressure). We note that the large line broadening $\left(F W H M \simeq 100-150 \mathrm{~km} \mathrm{~s}^{-1}\right)$ detected along portions of some of the jets of HLTau/HH30 complex (Mundt et al. 1990) is an indication that turbulent entrainment may be relevant in those regions, as suggested by Mundt et al.

We also performed short-time runs of adiabatic jets into stratified environments in order to compare their structure with that of radiative cooling jets (Figs 12 and 13). In general, the radiative cooling jet propagating into an ambient medium of positive density gradient is more collimated by the compressing medium than the adiabatic jet. On the other hand, the adiabatic jet propagating into an ambient medium of negative density gradient suffers more head decollimation. Both effects are due to the fact that the adiabatic jet has a higher pressure cocoon, which is better able to preserve the beam structure in the compressing environment, and to accelerate the ambient gas where its density is decreasing.

Finally, as in our previous investigations (GB93, GB94, CMGB94, GBB), in this study we have assumed a history-independent optically thin radiative cooling function to comput the losses of a fully ionized flow. By not following the history-dependent effects of nonequilibrium ionization of the gas or the transfer of ionizing radiation, we possibly underestimated the cooling rate in some parts of the postshock regions by as much as an order of magnitude (e.g., Innes, Giddings \& Falle 1987). The inclusion of a detailed cooling evaluation, as in the one-dimensional calculations of $\mathrm{HR}$, would require a substantial increase in computer power. Although future work should take into account those effects, we expect that the gross dynamical features obtained in the present analysis will not change. This expectation is supported by the comparison of our results (see GB93, GB94) 
with the calculations of SN (see also their recent calculations for steady flows; Stone \& Norman 1993b, 1994b), which included a nonequilibrium time-dependent cooling.

E.M.G.D.P. would like to acknowledge the very fruitful discussions with Willy Benz, Reinhard Mundt, and Tom Ray. The authors are also indebted to the referee for his relevant remarks. The simulations were performed on the Workstations HP apollo 9000/720 and Dec 3000/600 AXP of the Plasma Astrophysics Group of the Department of Astronomy of IAG/USP, whose purchase was made possible by the Brazilian agency FAPESP. This work was partially supported by a CNPq grant. 
TABLE 1 Values of the input parameters of our models.

\begin{tabular}{||c|c|c|c|c|c|c|}
\hline Figure & $\alpha$ & $\beta$ & $\eta$ & $M_{a}$ & $q_{b s}\left(x_{o}\right)$ & $q_{j s}\left(x_{o}\right)$ \\
\hline $1 \mathrm{a}$ & 0.5 & $5 / 3$ & 3 & 24 & 8.1 & 0.3 \\
$1 \mathrm{~b}$ & 0.1 & $5 / 3$ & 3 & 24 & 8.1 & 0.3 \\
1c & 0 & 0 & 3 & 24 & 8.1 & 0.3 \\
$1 \mathrm{~d}$ & 0.1 & $-5 / 3$ & 3 & 24 & 8.1 & 0.3 \\
$1 \mathrm{e}$ & 0.5 & $-5 / 3$ & 3 & 24 & 8.1 & 0.3 \\
$2,5 \mathrm{a}$ & 0 & 0 & 3 & 24 & 8.1 & 0.3 \\
$3,5 \mathrm{~b}$ & 0.5 & $5 / 3$ & 3 & 24 & 8.1 & 0.3 \\
$4,5 \mathrm{c}$ & 0.5 & $-5 / 3$ & 3 & 24 & 8.1 & 0.3 \\
$7 \mathrm{a}, 8$ & 0.5 & $5 / 3$ & 1 & 24 & 3.1 & 3.1 \\
$7 \mathrm{~b}$ & 0.5 & $5 / 3$ & 3 & 24 & 8.1 & 0.3 \\
$7 \mathrm{c}$ & 0.5 & $5 / 3$ & 10 & 24 & 16.7 & 0.008 \\
$9 \mathrm{a}$ & 0.5 & $-5 / 3$ & 1 & 24 & 3.1 & 3.1 \\
$9 \mathrm{~b}$ & 0.5 & $-5 / 3$ & 3 & 24 & 8.1 & 0.3 \\
$9 \mathrm{c}$ & 0.5 & $-5 / 3$ & 10 & 24 & 16.7 & 0.008 \\
10 & 0.5 & $5 / 3$ & 3 & 12 & 0.5 & 0.019 \\
11 & 0.5 & $-5 / 3$ & 3 & 12 & 0.5 & 0.019 \\
12 & 0.5 & $5 / 3$ & 3 & 24 & 0 & 0 \\
13 & 0.5 & $-5 / 3$ & 3 & 24 & 0 & 0 \\
\hline
\end{tabular}


TABLE 2 Velocity oscillations in the $\eta=3$ jet head of Fig. 3 (and Fig. 7b) propagating into an environment of increasing density $(\alpha=0.5, \beta=5 / 3)$.

\begin{tabular}{||c|c|c|c|c|}
\hline$t / t_{j a}$ & $\left(x_{b s}-x_{o}\right) / R_{j}$ & $(\epsilon)^{1 / 2}$ & $v_{b s}\left(k m s^{-1}\right)$ & $n_{a} v_{b s}^{2 \dagger}$ \\
\hline 0 & 0 & 1 & 250 & $2.3 \times 10^{2}$ \\
0.45 & 5.3 & 1.2 & 200 & $1.2 \times 10^{3}$ \\
0.85 & 8.8 & 1.4 & 144 & $1.2 \times 10^{3}$ \\
1.25 & 12 & 1.6 & 130 & $1.7 \times 10^{3}$ \\
1.65 & 18.7 & 3.3 & 260 & $1.2 \times 10^{4}$ \\
1.95 & 23 & 3.2 & 225 & $1.2 \times 10^{4}$ \\
\hline
\end{tabular}

${ }^{\dagger} \mathrm{n}_{a} \mathrm{v}_{b s}^{2}$ is in units of $\mathrm{n}_{a}\left(\mathrm{x}_{o}\right) \mathrm{c}_{a}^{2}=5.5 \times 10^{14} \mathrm{~cm}^{-1} \mathrm{~s}^{-2}$

TABLE 3 Velocity oscillations in the head of the $\eta=1$ jet of Fig. 7a propagating into an environment of increasing density $(\alpha=0.5, \beta=5 / 3)$.

\begin{tabular}{||c|c|c|c|c|}
\hline$t / t_{j a}$ & $\left(x_{b s}-x_{o}\right) / R_{j}$ & $(\epsilon)^{1 / 2}$ & $v_{b s}\left(k m s^{-1}\right)$ & $n_{a} v_{b s}^{2 \dagger}$ \\
\hline 0 & 0 & 1 & 199 & $1.4 \times 10^{2}$ \\
0.85 & 7.7 & 1.3 & 125 & $7.9 \times 10^{2}$ \\
1.25 & 13.1 & 6 & 224 & $5.3 \times 10^{3}$ \\
1.85 & 18.9 & 5 & 126 & $2.9 \times 10^{3}$ \\
\hline
\end{tabular}


TABLE 4 Velocity oscillations in the head of the $\eta=10$ jet of Fig. 7c propagating into an environment of increasing density $(\alpha=0.5, \beta=5 / 3)$.

\begin{tabular}{||c|c|c|c|c|}
\hline$t / t_{j a}$ & $\left(x_{b s}-x_{o}\right) / R_{j}$ & $(\epsilon)^{1 / 2}$ & $v_{b s}\left(k m s^{-1}\right)$ & $n_{a} v_{b s}^{2 \dagger}$ \\
\hline 0 & 0 & 1 & 302 & $3.3 \times 10^{2}$ \\
0.45 & 7.5 & 1.1 & 232 & $2.6 \times 10^{3}$ \\
0.65 & 10.5 & 1.7 & 249 & $4.8 \times 10^{3}$ \\
1.05 & 15.9 & 1.7 & 212 & $6.3 \times 10^{3}$ \\
1.25 & 19 & 2 & 259 & $1.2 \times 10^{4}$ \\
\hline
\end{tabular}




\section{REFERENCES}

Bally, J., Devine, D. 1994, Ap.J.(Lett.), 428, L65.

Benz, W.1990, in Numerical Modeling of Stellar Pulsations: Problems and Prospects, ed. J.R. Buchler (Dordrecht:Kluwer), p. 269.

Birkinshaw, M. 1991, in Beams and Jets in Astrophysics, ed. P.A. Hughes Cambridge, University Press, p. 278.

Biro, S. \& Raga, A.C. 1994

Blondin, J.M., Fryxell, B.A. \& Königl, A. 1990, Ap. J., 360, 370 (BFK).

Chernin, L., Masson, C., Gouveia Dal Pino, E.M. \& Benz, W. 1994, Ap. J., 426, 204 (CMGB).

Eislöffel J. 1993, Ph.D. Thesis, University of Heidelberg.

Falle, S.A.E.G. \& Raga, A.C. 1993, MNRAS, 261, 573.

Falle, S.A.E.G. \& Wilson, M.J. 1985a, MNRAS, 216, 79.

Falle, S.A.E.G. \& Wilson, M.J. 1985b, MNRAS, 216, 971.

Fuller, G.A., and Myers, P.C. 1992, Ap. J., 384, 523.

Gouveia Dal Pino, E.M. 1995, in Plasma Physics and Controlled Nuclear Fusion, eds. P. Sakanaka et al., AIP series (in press).

Gouveia Dal Pino, E.M. \& Benz, W. 1993, Ap. J., 410, 686 (GB93))

Gouveia Dal Pino, E.M. \& Benz, W. 1994, Ap. J., 435, 261 (GB94). 
Gouveia Dal Pino, E.M., Birkinshaw, M. \& Benz, W. 1996, Ap. J. (Lett.), 460, L111 (GBB).

Hardee, P.E. et al. 1992, Ap.J., 387, 460.

Hartigan, P., Mundt, R., and Stocke 1986, J., AJ, 91, 1357

Hartigan, P., \& Raymond, J. 1992, ApJ, 409, 705 (HR).

Hartigan, P., Raymond, J., \& Meaburn, J. 1990, ApJ, 362, 624.

Innes, D.E., Giddings, J.R., \& Falle, S.A.E.G. 1987, MNRAS, $226,67$.

Katz, J. 1989, Ph.D. thesis, Princeton University.

Kofman, L., \& Raga, A.C., 1992, ApJ, 390, 359 (KR).

López, R. et al. 1995, MNRAS (in press).

Morse, J. A. et al. 1992, ApJ, 339, 231.

Mundt, R. et al. 1984, A. A., 140, 17.

Mundt, R. et al. 1990, A. A., 232, 37.

Mundt, R., Brügel,E.W., \& Buhrke, T. 1987, ApJ, 319, 275.

Mundt, R., T.P. Ray, and Raga, A.C. 1991, A. A., 252, 740 (MRR).

Raga, A.C. 1993, Astrophys. and Space Sci., 208, 163., 258, 301.

Raga, A. C., Cabrit, S. and Cantó, J. 1994 (preprint).

Raga, A.C., Cantó, J., \& Biro, S. 1993, MNRAS, 260, 163.

Raga, A.C., \& Kofman, L., 1992, ApJ, 386, 222. 
Raga, A.C. \& Noriega-Crespo, A. 1993, Rev. Mex. A. Ap.,

Reipurth, B. 1989a, A. A., 220, 249.

Reipurth, B. 1989b, in ESO Workshop on Low Mass StarFormation and Pre-Main Squence Objects, ed. B. Reipurth, ESO, p. 247.

Reipurth, B., Raga, A.C. \& Heathcote, S. 1992, ApJ, 392, 145.

Sanders, R.H., 1983, ApJ, 266, 73.

Stahler, S. 1993 in Astrophysical Jets, ed. M. Livio, C.O.'Dea, \& D. Burgarella (Cambridge Univ. Press, p. 183.

Stone, J.M., \& Norman, M.L. 1993a, ApJ, 413, 210 (SN).

Stone, J.M., \& Norman, M.L. 1993b, ApJ, 413, 198.

Stone, J.M., \& Norman, M.L. 1994a, ApJ, 420, 237.

Stone, J.M., \& Norman, M.L. 1994b, ApJ, 422, 348.

Wiita P.J, Rosen, A. and Norman, M.L. 1990, ApJ, 350, 545. 


\section{Figure Captions}

Figure 1. Mid-plane density contours and velocity distribution of five radiative cooling jets at $\mathrm{t}=0.85 R_{j} / c_{a}(=38.2 \mathrm{yr})$ evolving through different stratified ambient media with: a) $\alpha=0.5, \beta=5 / 3$, b) $\alpha=0.1, \beta=5 / 3$, c) $\alpha=0, \beta=0$, d) $\alpha=0.1, \beta=-5 / 3$, and e) $\alpha=0.5, \beta=-5 / 3$. The initial parameters are $\eta=3, n_{a}=200 \mathrm{~cm}^{-3}, R_{j}=2 \times 10^{15} \mathrm{~cm}$, $M_{a}=24$, and $v_{j}=398 \mathrm{~km} / \mathrm{s}$. The $\mathrm{z}$ and $\mathrm{x}$ coordinates are in units of $R_{j}$. The contour lines are separated by a factor of 1.3 and the density scale covers the range from $\simeq 0.02$ up to $363 / n_{a}$. The maximum density reached by the cold shell developed at the head of the jet is $n_{s h} / n_{a}\left(x_{o}\right) \approx:$ a) 363 , b) 294 , c) 197 , d) 69 , and e) 32 .

Figure 2. Mid-plane density contour and velocity distribution evolution of a radiative cooling jet propagating into a homogeneous ambient medium $(\alpha=0, \beta=0)$. The initial conditions are the same as in Fig. $1\left(\eta=3, n_{a}=200 \mathrm{~cm}^{-3}, R_{j}=2 \times 10^{15} \mathrm{~cm}, M_{a}=24\right.$, and $v_{j}=398 \mathrm{~km} / \mathrm{s}$ ). The contour lines are separated by a factor of 1.3 and the density scale covers the range from $\simeq 0.16$ up to $400 / n_{a}$. The times (in units of $t_{j a}=R_{j} / c_{a}=38.2$ yr) and jet head positions depicted are: a) $0.70 t_{j a}$ and $10.6 R_{j}$; b) $1.05 t_{j a}$ and $17.6 R_{j}$; c) $1.40 t_{j a}$ and $24.6 R_{j}$; and d) $1.75 t_{j a}$ and $31.6 R_{j}$. The maximum density of the cold shell at the head, $n_{s h} / n_{a}\left(x_{o}\right)$, is: $\approx$ a) 195, b) 400, c) 238 , and d) 272 .

Figure 3. Mid-plane density contour and velocity distribution evolution of a radiative cooling jet propagating into an ambient medium with positive density (and pressure) gradient $(\alpha=0.5, \beta=5 / 3)$. The initial conditions are the same as in Fig. 2. The contour lines are separated by a factor of 1.2 and the density scale covers the range from $\simeq 0.01$ up

to $3550 / n_{a}$. The times and jet head positions are: a) $0.85 t_{j a}$ and $8.8 R_{j}$; b) $1.25 t_{j a}$ and 12 $R_{j}$; c) $1.65 t_{j a}$ and $18.7 R_{j}$; and d) $1.96 t_{j a}$ and $23 R_{j}$.

Figure 4. Mid-plane density contour and velocity distribution evolution of a radiative 
cooling jet propagating into an ambient medium with negative density (and pressure) gradient $(\alpha=0.5, \beta=-5 / 3)$. The initial conditions are the same as in Fig. 2. The contour lines are separated by a factor of 1.2 and the density scale covers the range from $\simeq 0.01$ up to $29 / n_{a}$. The times and jet head positions are: a) $0.25 t_{j a}$ and $4.3 R_{j}$; b) $0.65 t_{j a}$ and 12.3 $R_{j}$; c) $1.05 t_{j a}$ and $20.8 R_{j}$; and d) $1.45 t_{j a}$ and $30 R_{j}$. The maximum density constrast in the thin shell at the head is $n_{s h} / n_{a}\left(x_{o}\right) \approx 13,23,31$, and 18, in Figs. 4a, b, c, and d, respectively.

Figure 5. Mid-plane radiative emissivity contour of the jets of Figs. 2, 3, and 4 when they reach $x \simeq 25 R_{j}$. a) jet in the homogeneous ambient medium $(\alpha=0, \beta=0)$ at $\mathrm{t}$ $\left.=1.4 t_{j a} ; \mathrm{b}\right)$ jet in the increasing density ambient medium $(\alpha=0.5, \beta=5 / 3)$ at $\mathrm{t}=1.96$ $t_{j a}$; c) jet in the decreasing density ambient medium $(\alpha=0.5, \beta=-5 / 3)$ at $\mathrm{t}=1.25 t_{j a}$. The emissivity is in units of $3.8 \times 10^{-19} \mathrm{erg} \mathrm{cm}^{-3} \mathrm{~s}^{-1}$. The contour lines are separated by a factor of 1.2 and the emissivity scale covers the range from $\simeq 0.01$ up to $3.3 \times 10^{5}$ in code units.

Figure 6. Time evolution of the axial radiative emissivity of the head of the jets of Figs. $2(\alpha=0, \beta=0), 3(\alpha=0.5, \beta=5 / 3)$, and $4(\alpha=0.5, \beta=-5 / 3)$. The emissivity is in units of $3.8 \times 10^{-19} \mathrm{erg} \mathrm{cm}^{-3} \mathrm{~s}^{-1}$.

Figure 7. Velocity distribution of the mid-plane of cooling jets with different $\eta$ propagating into an $\alpha=0.5$ ambient medium with positive $(\beta=5 / 3)$ density (and pressure) stratification: a) $\eta=1$ : b) $\eta=3$ : and c) $\eta=10$. The corresponding density contours are shown in Fig. 2 of GBB. The other initial conditions are the same of the previous figures. The contour lines are separated by a factor of 1.3 and the density scale covers the range from $\simeq 0.01$ up to $2540 / n_{a}$. The $\eta=1$ reaches the end of the computation domain at $t=1.85$ $t_{j a}$; the $\eta=3$ at $t=1.65 t_{j a}$ and the $\eta=10$ jet at $t=1.25 t_{j a}$. 
Figure 8. Mid-plane density contour and the corresponding velocity distribution of the $\eta=1$ jet of Fig. 7 at $t=2.05 t_{j a}$. The contour lines are separated by a factor of 1.3 and the density scale covers the range from $\simeq 0.05$ up to $205 / n_{a}$. The knots close to the head seem to break and separate apart.

Figure 9. Mid-plane density contour and the corresponding velocity distribution of cooling jets with different $\eta$ propagating into an $\alpha=0.5$ ambient medium with negative $(\beta=-5 / 3)$ density (and pressure) stratification and the same initial conditions of Fig. 5: a) $\eta=1$ : b) $\eta=3$ : and c) $\eta=10$. The jets reach the end of the domain, at $x \approx 20 R_{j}$, at $t=$ :a) $1.05 t_{j a}(\eta=1)$; b) $0.95 t_{j a}(\eta=3)$; and c) $0.85 t_{j a}(\eta=10)$. The contour lines are separated by a factor of 1.3 and the density scale covers the range from $\simeq 0.01$ up to $58 / n_{a}$. The maximum density in the cold shell at the head is $n_{s h} / n_{a}\left(x_{o}\right) \approx 4.4,33$, and 58 , respectively.

Figure 10. Mid-plane density contour and velocity distribution evolution of an $\eta=3$ cooling jet propagating into an $\alpha=0.5$ ambient medium with positive $(\beta=5 / 3)$ density (and pressure) stratification and $M_{a}=12$ ( or $v_{j}=199 \mathrm{~km} \mathrm{~s}^{-1}$ ). The other initial conditions are the same of Fig. 3, except that $q_{b s}\left(x_{o}\right) \approx 0.5$ (eq. 5) and $q_{j s}\left(x_{o}\right) ; \approx 1.9 \times 10^{-2}$. The contour lines are separated by a factor of 1.3 and the density scale covers the range from $\simeq 0.01$ up to $284 / n_{a}$. The times depicted are: a) $1.15 t_{j a}$; b) $2.45 t_{j a}$; and c) $3.85 t_{j a}$.

Figure 11. Mid-plane density contour and velocity distribution evolution of an $\eta=3$ cooling jet propagating into an $\alpha=0.5$ ambient medium with negative $(\beta=5 / 3)$ density (and pressure) stratification and $M_{a}=12\left(\right.$ or $\left.v_{j}=199 \mathrm{~km} \mathrm{~s}^{-1}\right)$. The other initial conditions are the same of Fig. 4 except that $q_{b s}\left(x_{o}\right) \approx 0.5$ (eq. 5) and $q_{j s}\left(x_{o}\right) ; \approx 1.9 \times 10^{-2}$. The contour lines are separated by a factor of 1.3 and the density scale covers the range from $\simeq 0.01$ up to $14 / n_{a}$. The times depicted are: a) $0.65 t_{j a}$; b) $1.25 t_{j a}$; and c) $1.85 t_{j a}$. The 
jet reaches the end of the domain at $x \approx 20 R_{j}$. The maximum density in the cold shell is $n_{s h} / n_{a}\left(x_{o}\right) \approx 14,11$, and 6 in the successive frames of the Figure.

Figure 12. Central density contour and velocity distribution of an adiabatic (a) and a radiative cooling (b) jet, both with $\eta=3$ and $M_{a}=24$ propagating into an $\alpha=0.5$ ambient medium with positive $(\beta=5 / 3)$ density (and pressure) stratification at $t=1.45 t_{j a}$. The contour lines are separated by a factor of 1.3 and the density scale covers the range from $\simeq 0.01$ up to $650 / n_{a}$.

Figure 13. The density contours and velocity distribution of an adiabatic (a) and a radiative cooling (b) jet, both with $\eta=3$ and $M_{a}=24$ propagating into an $\alpha=0.5$ ambient medium with negative $(\beta=-5 / 3)$ density (and pressure) stratification at $t=0.85 t_{j a}$ when they reach $\mathrm{x} \approx 20 R_{j}$. The contour lines are separated by a factor of 1.3 and the density scale covers the range from $\simeq 0.01$ up to $32 / n_{a}$. 